\title{
Utilising Epidemiological Dynamics of SARS-CoV-2 Cases in Nigeria to Predict the Dis- semination Pattern in Different States and Local Government Areas
}

\author{
Mary A. Oboh ${ }^{1 *}$, Seun O. Agbanah-Owa ${ }^{2}$, Ifeoluwa K. Fagbohun ${ }^{3}$, Olumide Ajibola ${ }^{1,4}$ \\ ${ }^{1}$ Medical Research Council Unit, the Gambia at the London School of Hygiene and Tropical Medicine. \\ ${ }^{2}$ Providence Research LTD, Ibadan, Nigeria. \\ ${ }^{3}$ Department of Zoology, Parasitology and Bioinformatics Unit, University of Lagos, Nigeria. \\ ${ }^{4}$ First Technical University, Ibadan Oyo State
}

\begin{abstract}
*Correspondence should be addressed to Mary A. Oboh: moboh@mrc.gm
Received 25th May 2021; Revised 13th July 2021; Accepted 15September 2021

(C) 2021 Oboh et al. Licensee Pan African Journal of Life Sciences an official publication of Faculty of Basic Medical Sciences, Ladoke Akintola University of Technology, Ogbomoso. This is an Open Access article distributed under the terms of the Creative commons Attribution License (https://creativecommons.org/licenses/BY/4.0), which permits unrestricted use, distribution, and reproduction in any medium, provided the original work is properly cited.
\end{abstract}

\begin{abstract}
Background: More than a year following the emergence of severe acute respiratory syndrome coronavirus 2 (SARS-CoV-2), the effect on health, economics, travel, and social aspects of life remains evident. With the deployment of the coronavirus disease 2019 (Covid-19) vaccine to various countries, it is believed that the impact of Covid-19 on populations will be mitigated. However, due to vaccine shortage and uncertainty about the duration of protection, individuals must adhere to effective preventive measures that could reduce the spread of the virus. This is important as countries begin to open their borders. Using the susceptible, exposed, infected, and recovered model, we set out to estimate the incidence rate and spread of COVID-19 to the different Local Government Areas (LGAs).
\end{abstract}

Methods: In this study, we collected SARS-CoV-2 incidence data generated by the Nigerian Centre for Disease Control (NCDC) from the onset of the pandemic until October 2020 to retrospectively predict the incidence at the beginning of the pandemic in Nigeria.

Results: We observed discrepancies in incidence between our model and the NCDC COVID-19 cases at different time points resulting in a weak positive correlation. Unfortunately, it was observed from our model that SARS-CoV-2 will still be in circulation even in 2022.

Conclusion: While the government of Nigeria must open up the borders, appropriate preventive measures and massive vaccine campaigns to increase vaccine coverage should be in place.

Keywords: Severe Acute Respiratory Syndrome Coronavirus 2, Nigeria, SEIR model, Covid-19 cases, vaccine 


\subsection{INTRODUCTION}

Since the middle of December 2019, the novel severe acute respiratory syndrome coronavirus 2 (SARS-CoV-2) or coronavirus disease 2019 (COVID-19), which originated in China, has spread rapidly to almost all countries of the globe. Several months into the COVID19 pandemic, the number of cumulative cases remains on the upload slope [1], especially in the global north, resulting in unparalleled pressure on health care systems and global economies, causing staggering deaths $[2,3]$. Several preventive and control measures have been adopted in response to this global pandemic, such as physical distancing, hand hygiene, wearing of nose masks and face shields. Over a hundred vaccine candidates are currently at different stages of clinical trials, with some currently approved for usage in different countries of the world [4-6]. Thus, all efforts at containing the spread have resulted in substantial economic impacts on countries, especially those in resource-limited settings.

As most countries attempt to return to normalcy due to the COVID-19 pandemic, most low- and middle-income countries (LMICs) are still seeking evidence-based policy guidance on easing the lockdown as the numbers of cases appear to be on the decline. Nigeria has used a phased approach in relaxing some of the restrictions placed on most sectors of the economy due to a significant reduction in the number of COVID-19 cases and death. However, there is no evidence to suggest that relaxing restrictions was based on projections from empirical data.

The Nigerian Centre for Disease Control has been at the forefront of controlling the spread and transmission of SARS-CoV-2 with a tremendous effort in testing, isolation, contact tracing, and management of COVID-19 infected patients. Further, they have provided updates of infection rates across the country on a real-time basis.

At the onset of any epi-/pandemic, policymakers rely significantly on estimates of transmissibility and reproductive numbers to predict the spread of the infection, aiming to mitigate further spread of the causative agent. Therefore, it is imperative to consider all factors and variables necessary for reopening the country using scientific data, as this will guide the country in a clear direction. The susceptible-exposed-infectedrecovered (SEIR) compartment model has been used to estimate the peak of the coronavirus disease in Japan [7]; reproductive number of SARS-CoV-2 [8]; impacts of seasonal dynamics on the transmissibility of SARS-CoV$2[9]$.

To this end, we utilised the SEIR compartment model that factors in variables including general demographic data, the trend of COVID-19 cases, mortalities, recoveries to estimate the incidence rate and spread of COVID-19 to the different local government areas (LGAs), taking into cognizance various scenarios. Thus, we asked the question i). How has the population size, industrial mobility, and cultural differences impacted the total reopening of the Nigerian economy following SARS-CoV-2 pandemic?

\subsection{METHODOLOGY}

\subsection{Acquisition of Data}

Incidence of SARS-CoV-2 per state per month (From March - September 2020) was obtained from the Nigeria Centre for Disease Control (NCDC) (Supplementary Table 1). The data used for our model is publicly available at https://ncdc.gov.ng

\subsection{Model}

The Susceptible, Exposed, Infected, Recovered (SEIR) Compartmental Model built $[10,11]$ on The Spatiotemporal Epidemiological Modeler (STEM) was applied to explore the progression of the virus and predict the outcomes of SARS-CoV-2 infection in Nigeria from February 27th, 2020 to February 29th, 2024. This model explores the possible outcomes of different ways of managing the spread of SARS-CoV-2 infection by simulating the following two scenarios: (i) the base scenario in which COVID-19 takes its natural cause with no measure to control it and (ii) a combined Social distancing and lockdown scenario. Thus, the model created to represent Nigeria accounts for Human population, road interconnectedness, air traffic, and the local government.

\subsection{Scenarios}

\subsubsection{Base Scenario}

The Infector (representing the index case) was introduced on February 27th, 2020, and the model was allowed to run till February 29th, 2024. A logger was attached to the model to capture the model state. 


\subsubsection{Social Distancing and Lockdown}

The effect of social distancing and lockdown is incorporated into the same model by introducing a trigger that alters the infection rate to account for the effect of social distancing introduced into the model 57 days after the index case was observed. Also, the lockdown was modelled by introducing a trigger to alter the mobility of people. This trigger was introduced into the model 57 days after the index case was observed and lifted 95 days later.

\subsection{Assumptions made During the Modelling:}

1. It was assumed that the lone index case was introduced from the Murtala Mohammed International Airport in Lagos.

2. Lockdown was $70 \%$ effective in reducing SARS-CoV2 transmission as the intra-state movement was still ongoing during the national lockdown.

3. Population in the year 2020 was projected from the census population in year 2006 by taking into account population growth rates at 0.027-0.093.

\subsection{Model creation}

The model was created by assembly from pre-existing components in SEIR, including human population, physical boundaries, road network, air traffic, and disease model $[10,11]$. The pre-existing models of the human population in STEM capture the population data for the year 2006. This was updated for population growth using average populating growth rates by local government areas. Thus, the model created for the simulation reflects the prevailing population in Nigeria in the year 2020

\subsection{RESULTS}

The estimated distribution and incidence of COVID-19 infection as of March 30th 2020 is presented in Figure 1. At the beginning of the spread of SARS-CoV-2 cases in Nigeria, our model estimated 36 cases while the NCDC reported 20 confirmed cases during the same period. The output from our model suggested widespread distribution across different states, therefore becoming almost negligible relative to the population proportion as observed from the map (attack rates).

This study showed that the spread of SARS-CoV-2 as early June 2020 in Nigeria originated mainly from Lagos

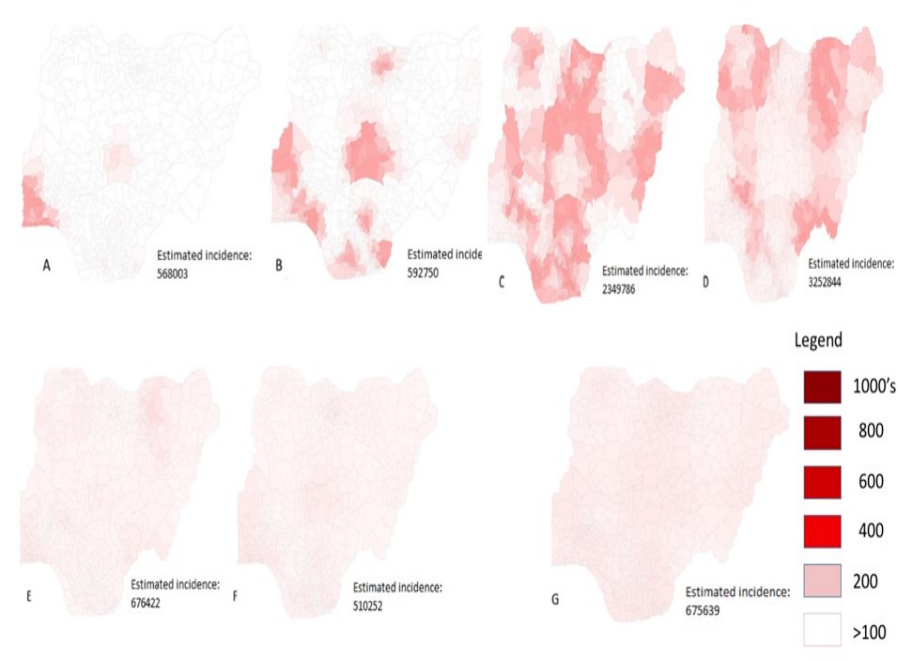

Figure 1. Estimated incidences of SARS-CoV-2 from the model in (a) June: infection originating mainly from Lagos and Abuja (b) July: when the virus was gradually being transmitted to various states (c) August (d) September: SARS-CoV-2 already circulating in almost all parts of the country (e) October (f) November (g) December: saturation of infection in the entire country). All months represented here are for 2020.

Colour intensity on figure represent the estimated number of SARS -CoV-2 cases in tens, hundreds and thousands in the different regions.

and Abuja; where two of the five countries' functional international airports are located (Figure 1a). As the pandemic progressed, the virus continued to spread to various parts of the country with varying intensity of infection (Figure 1b) and established its prevalence more in the southwestern, southeastern, northcentral northwestern regions of the country. However, by August/September 2020, the infection had spread throughout the country again with varying infection rates (Figures 1c and 1d).

The national lockdown was between March 30th to May 4th, 2020, and this might have reduced the rate of the spread of the virus, as evident in the fact that the virus spread (Figure 1e-g) did not reach saturation until the end of the year 2020 .

Our study predicted that although there will be a continuous decrease in the national incidence of COVID19 (from the comparison between incidence from January and February 2021 to that of January and February 2022), this decrease, however, will not be a drastic one as there will still be the circulation of SARSCoV-2 within the country (Figure 2A-D).

The differences between the actual confirmed cases (from the NCDC website) and simulated cases from this 
study were between $97-99 \%$. We predicted that this may have been due to the low coverage of COVID-19 testing in Nigeria. Consequently, a weak positive correlation between the confirmed active cases (as reported by NCDC) and the simulated incidence (Figure 3). Therefore, each (NCDC confirmed and simulated cases) may not be a good predictor of each other. This could be attributed to the unpredictability of human behaviour, which has a significant impact on the transmission of the disease. A similar study in Italy had reported much lower unreported cases of $72 \%$ [12].

The relaxation of the national lockdown is believed to have led to the spike in COVID-19 incidence in the country as a sudden continuous increase was recorded from May 2020, reaching its peak in August 2020 before a steady decline (Figure 4) was observed. Similarly, this same trend was noted in the daily reports by the NCDC.

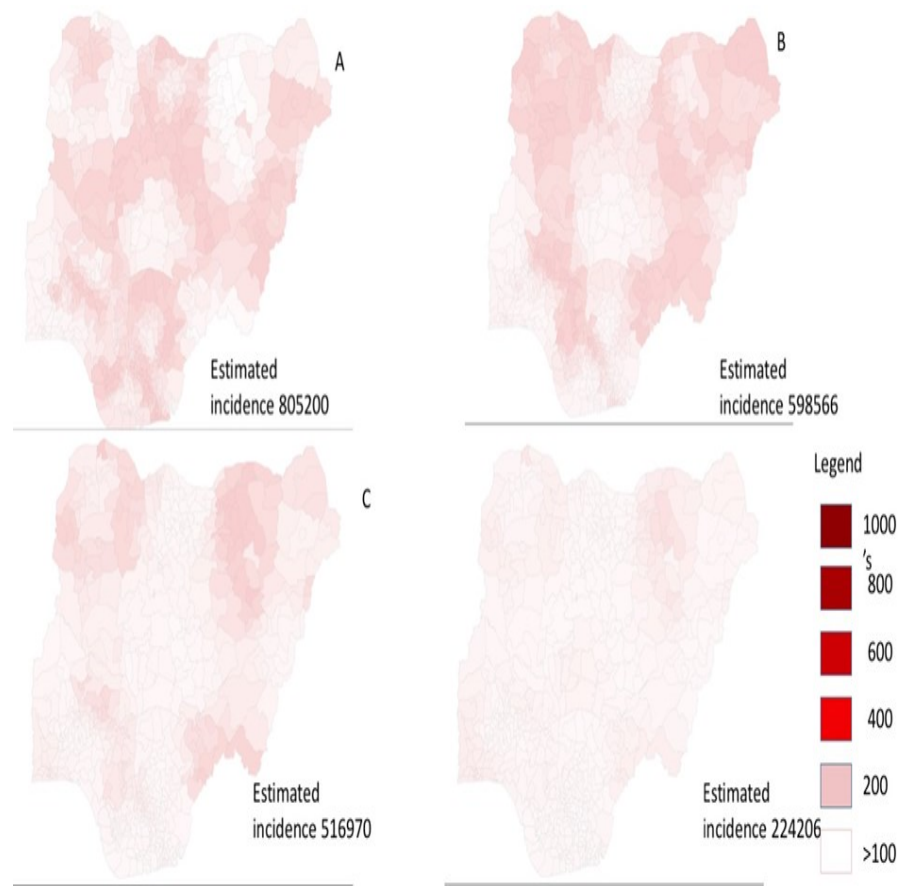

Figure 2: Gradual decrease of SARS-CoV-2 (a) January 2021 (b) February 2021 (c) January, 2022 (d) February, 2022. All months represented here are for 2020.

Colour intensity on figure represent the estimated number of SARS-CoV-2 cases in tens, hundreds and thousands in the different regions

\subsection{DISCUSSION}

The first case of the novel coronavirus disease in Nigeria was recorded on the 27th of February 2020. Since then, the virus has spread to different parts of the country with varying levels of infection intensity. COVID-19 testing in
Nigeria is limited [13] and may not have given an accurate picture of the spread and prevalence of the infection in the country. Our SEIR model seeks to provide an estimated incidence rate and spread of COVID-19 to the different LGAs taking into cognizance various scenarios described above. Due to lack of vaccine and standard control measures and drugs during the initial phase of the pandemic, COVID-19 control was restricted to basic hygiene practices, physical distancing, and use of personal protection equipment, i.e., nose masks.

Although, previous studies have reported the importance of international air travels in enhancing the spread of the virus $[12,13]$, as was also observed in the spread of

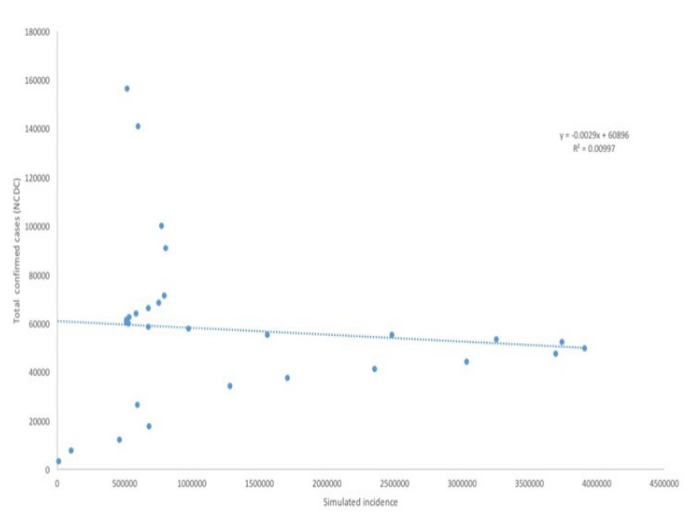

Figure 3: Correlation between NCDC confirmed COVID-19 cases and simulated cases in Nigeria.

SARS-CoV-2 in Nigeria from two key states; Lagos and

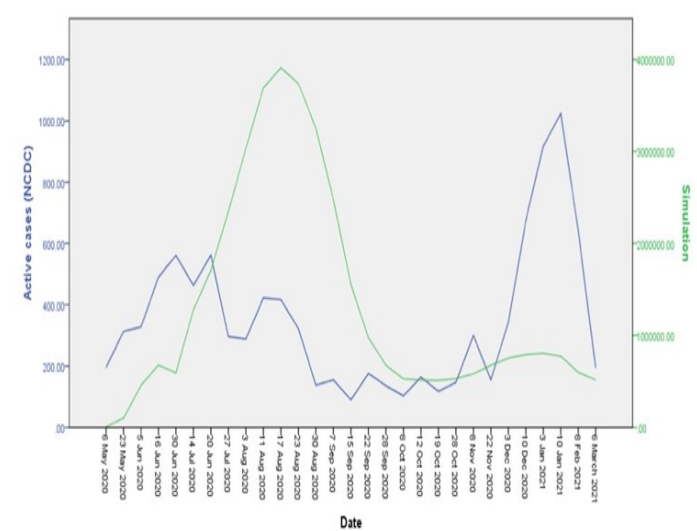

Figure 4. Incidence of simulated and NCDC confirmed COVID-19 cases in Nigeria. Peak incidence observed in August 2020 from both data source. 
Abuja (where two of the five country's functional international airport are located). However, the spread was not rapid, perhaps due to restriction of international flights into Nigeria, mainly through the airports in Lagos and Abuja.

Although the virus spread throughout the country's different geopolitical zones, from the southwestern, southeastern, north-central to the northwestern regions of the country, infection rate was not saturated in the country until the end of the year 2020. Thus, it could be predicted that the national lockdown could have mitigated the spread of SARS-CoV-2, albeit for a brief period.

We observed a $97-99 \%$ difference between NCDC confirmed cases and our model estimated cases. This variation could be due to the low coverage of COVID-19 testing in Nigeria, where only 2362757 tests have been done in a population of over 2 million [14]; a similar trend has been observed in different African countries [15]. This peripherally could be seen as a low prevalence of SARS-CoV-2 in Africa while undetected and hidden transmission is ongoing. Moreover, this difference could also be attributed to the unpredictability of human behaviour, which has a massive impact on the transmission of the disease. A similar study in Italy had reported much lower unreported cases of $72 \%$ [12].

As shown from our model, SARS-CoV-2 will likely remain in Nigeria and the world for a longer than anticipated time. Thus, to promote a safe and effective gradual recovery of international travel while managing the public health risks associated with the cross-border movement of people and goods, key stakeholders (including the Member States, the travel industry and its affiliates, and the general public) should in addition to receiving the vaccination adhere to using personal protection equipment and physical distancing.

\section{Acknowledgement}

The authors are grateful to the Nigerian Centre for Disease Control

\section{Conflict of interest}

The Author declare that there is no conflict of interest

\section{Authors' Contributions}

MAO conceived and designed the study, contributed to data collection and manuscript writing. SOA-O and
IKF performed data analysis and contributed to manuscript writing. OA performed manuscript critique and review All authors approved the final copy of the manuscript.

\section{References}

1. World Health Organisation. Coronavirus Disease (COVID-19) Situation Report- 195. 2020. doi:10.1213/xaa.0000000000001218.

2. World Health Organisation. Coronavirus disease 2019 (COVID-19) Situation Report - 90. 2020. doi:10.1001/jama.2020.2633.

3. McKee M, and Stuckler D. If the world fails to protect the economy, COVID-19 will damage health not just now but also in the future. Nat Med. 2020. 26:640-2. doi:10.1038/s41591-020-0863-y.

4. Kim JH, Marks F, and Clemens JD. Looking beyond COVID-19 vaccine phase 3 trials. Nat Med. [Accessed: 2021.27, 205-211]

5. Haque A, and Pant AB. Efforts at COVID-19 Vaccine Development: Challenges and Successes. Basel. December 1-16.2020. 8(4): 739

6. Bollyky TJ, Relations F, Gostin LO, Law H, and Hamburg MA. The Equitable Distribution of COVID -19 Therapeutics and Vaccines. 2020. 323:2020-1.

7. Kuniya T. Prediction of the Epidemic Peak of Coronavirus Disease in Japan, 2020. J Clin Med. 2020. 9:789.

8. Zhou T, Liu Q, Yang Z, Liao J, Yang K, Bai W, Xin, $\mathrm{L}$, and Wei, Z. Preliminary prediction of the basic reproduction number of the Wuhan novel coronavirus 2019-nCoV. J Evid Based Med. 2020. 13:3-7.

9. Neher RA, Dyrdak R, Druelle V, Hodcroft EB, and Albert J. Potential impact of seasonal forcing on a SARS-CoV-2 pandemic. Swiss Med Wkly. 2020. 150 March:w20224.

10. Wirawan IA, and Januraga PP. Forecasting COVID19 Transmission and Healthcare Capacity in Bali , Indonesia. J Prev Med Public Heal. 2020. 53:158-63.

11. Mbuvha R, and Marwala T. Bayesian Inference of COVID-19 Spreading Rates in South Africa Methods Epidemiological Modelling. PLoS ONE. 2020.15 (8):e0237126 
12. Tuite AR, Ng V, Rees E, and Fisman D. Estimation of COVID-19 outbreak size in Italy. Lancet Infect Dis. 2020. 20:537. doi:10.1016/S1473-3099(20) 30227-9.

13. Adegboye OA, Adekunle AI, and Gayawan E. Early transmission dynamics of novel coronavirus (Covid$19)$ in Nigeria. Int J Environ Res Public Health. 2020. 17(9):3054
14. Nigeria Centre for Disease Control (NCDC). NCDC Coronavirus COVID-19 Microsite. 2021. https:// covid19.ncdc.gov.ng. Accessed 13 Jul 2021.

15. Adebisi YA, Oke GI, Ademola PS, Chinemelum IG, and Ogun IO. SARS-CoV-2 diagnostic testing in Africa : needs and challenges. Pan Afr Med J. 2020. 35 (supp 2):4. 